\title{
Laminated currents
}

\author{
JOHN ERIK FORNÆSS $†$, YINXIA WANG $\ddagger$ and ERLEND FORNÆSS WOLD§ \\ $\dagger$ Mathematics Department, The University of Michigan, East Hall, Ann Arbor, \\ MI 48109, USA \\ (e-mail:fornaess@umich.edu) \\ \$ Department of Mathematics, Henan Polytechnic University, Jiaozuo, 454000, China \\ (e-mail: yinxiawang@gmail.com) \\ $\S$ Mathematisches Institut, Universität Bern, Sidlerstr. 5, CH-3012 Bern, Switzerland \\ (e-mail: erlendfw@math.uio.no)
}

(Received 9 March 2007 and accepted in revised form 26 October 2007)

Abstract. In this paper we prove the equivalence of two definitions of laminated currents.

\section{Introduction}

Let $K$ be a relatively-closed subset of the bidisc $\Delta^{2}(z, w)=\{(z, w) ;|z|,|w|<1\}$. We suppose that $K$ is a disjoint union of holomorphic graphs, $w=f_{\alpha}(z)$, where $f_{\alpha}$ is a holomorphic function on the unit disc with $f_{\alpha}(0)=\alpha$ and $\left|f_{\alpha}(z)\right|<1$. We let $\mathcal{L}$ denote the lamination of $K$.

There are two notions of laminated currents that we will discuss. Let $T$ be a positive closed $(1,1)$-current supported on $K$. We assume that $T$ is the restriction of a positive closed current defined on a neighborhood of $\bar{\Delta}^{2}$. We denote by $\left[V_{\alpha}\right]$ the current of integration along the graph of $f_{\alpha}$. Let $\lambda$ denote a continuous $(1,0)$-form which at $\left(z, f_{\alpha}(z)\right)$ equals a non-zero multiple of $d w-f_{\alpha}^{\prime}(z) d z$.

Definition 1. We say that $T$ is a laminated current directed by $\mathcal{L}$ if $\lambda \wedge T=0$ for any such $\lambda$.

These are the same as Sullivan's structure currents [10]. The present terminology was introduced by Berndtsson and Sibony in [1], and such currents were treated further in [4]. In accordance with Dujardin [3] we also define the following.

Definition 2. We say that $T$ is a laminated current subordinate to $\mathcal{L}$ if there is a positive measure $\mu$ such that $T=\int_{\alpha}\left[V_{\alpha}\right] d \mu(\alpha)$.

Our main result is the following.

MAIN THEOREM. The current $T$ is subordinate to $\mathcal{L}$ if and only if it is directed by $\mathcal{L}$. 
We note that this is a result by Sullivan in the case of the lamination being smooth, i.e. the graphs vary smoothly with $\alpha[\mathbf{1 0}]$. In the continuous setting Dujardin has shown that if a current $T$ is dominated by a current subordinate to $\mathcal{L}$ then $T$ is subordinate to $\mathcal{L}$.

The part of Sullivan's proof that does not go through automatically in the non-smooth case is a certain approximation step, and so in the present article we are concerned with approximation of partially-smooth functions. In [5] the authors proved such an approximation theorem in the case of laminations in $\mathbb{R}^{2}$ and in $\mathbb{R}^{3}$. In the last section we show that the main theorem breaks down for Riemann-surface laminations in higher dimension.

For related material on laminated currents the reader may consult the paper of Bedford et al [2].

\section{Holomorphic motions and preliminary estimates for slopes of holomorphic graphs}

We need to know how the lamination $\mathcal{L}$ defined above varies with the parameter $\lambda$, and we use the fact that it defines a holomorphic motion. Let $\Delta:=\{z \in \mathbb{C}:|z|<1\}$ denote the unit disc in $\mathbb{C}$. A holomorphic motion is a subset $E$ of the complex plane $\mathbb{C}$ (or the Riemann sphere $\widehat{\mathbb{C}}$ ) and a map $f: \Delta \times E \rightarrow \mathbb{C}$ (or $\widehat{\mathbb{C}}$ ) such that $f(0, \cdot)=$ id, $f(\lambda, \cdot)$ is injective for each $\lambda$, and $f(\cdot, z)$ is holomorphic for each $z$. The lamination $\mathcal{L}$ defines a holomorphic motion.

Let us briefly recall some facts. It is known [9] that any holomorphic motion has an extension to a holomorphic motion $f: \Delta \times \mathbb{C} \rightarrow \mathbb{C}$. This means that we may regard $K$ as a subset of a lamination of $\Delta \times \mathbb{C}$. From [8] we have that $f$ is automatically jointly continuous in $(\lambda, z)$; in fact the map $(\lambda, z) \mapsto\left(\lambda, f_{\lambda}(z)\right)$ is a homeomorphism onto $\Delta \times \mathbb{C}$. Moreover, $f(\lambda, \cdot)$ is quasi-conformal for each $\lambda$, and $f(\lambda, \cdot)$ distorts cross-ratios by a bounded amount depending on $|\lambda|$. In particular we have the following. If $C$ is compact in $\mathbb{C}^{*}$ and $x, y, z$ are three distinct points in $\mathbb{C}$ with $c_{0}=(x-y) /(z-y) \in C$, then $\left(f_{\lambda}(x)-f_{\lambda}(y)\right) /\left(f_{\lambda}(z)-f_{\lambda}(y)\right)$ is close to $c_{0}$ depending only on $|\lambda|$ (for a fixed $C)$. To see this one can consider the map $\lambda \mapsto\left(f_{\lambda}(x)-f_{\lambda}(y)\right) /\left(f_{\lambda}(z)-f_{\lambda}(y)\right)$, a map from the unit disk to $\mathbb{C} \backslash\{0,1\}$, and use the fact that it has to be distance-decreasing in the Poincaré metric. Finally we recall that $f(\lambda, \cdot)$ is Hölder continuous with exponent $1+\epsilon(|\lambda|)$.

Next we need a basic estimate on slopes of the graphs. For the benefit of the reader we include the details of this well-known fact. We denote by $\mathcal{O}(\Omega)$ the space of holomorphic functions on $\Omega$. Let $\|\cdot\|_{\infty}$ denote the sup norm. Set

$$
H^{\infty}=H^{\infty}(\Delta)=\left\{f \in \mathcal{O}(\Delta):\|f\|_{\infty}<\infty\right\} .
$$

Also, if $0<C<\infty$ we set

$$
H_{C}^{\infty}=H_{C}^{\infty}(\Delta)=\left\{f \in \mathcal{O}(\Delta):\|f\|_{\infty}<C\right\} .
$$

LEMMA 1. If $f \in H_{1}^{\infty}(\Delta)$ and $f(z) \neq 0$ for all $z \in \Delta$, then

$$
\left|f^{\prime}(0)\right| \leq 2|f(0)| \log (1 /|f(0)|) \text {. }
$$

Proof. Pick a holomorphic function $f(z)$ on the unit disc such that $0 \neq|f(z)|<1$ for all $z \in \Delta$. We can replace $f(z)$ by $e^{i \theta} f(z)$ for any real $\theta$. This does not change $|f(0)|$ or $\left|f^{\prime}(0)\right|$. Hence we can assume that $f(0)>0$. 
We set $h(z):=\log f(z)$. Then $h(z)$ is a holomorphic function on the unit disc and $\operatorname{Re}(h(z))<0$. We can also choose a branch of the logarithm so that $\log (f(0))=-a<0$. If $k(z)=h(z) / a$, then $k(z)$ is a holomorphic function on the unit disc and $k(0)=-1$, $\operatorname{Re}(k(z))<0$. We define $L(w)=(w+1) /(w-1)$. Then $L(-1)=0$ and if $\operatorname{Re}(w)<0$ then $|L(w)|<1$. Then $\Gamma(z):=L(k(z))$ is a holomorphic function from the unit disc to the unit disc. Moreover $\Gamma(0)=L(k(0))=L(-1)=0$. Since $\Gamma(0)=0$ and $|\Gamma(z)|<1$ we can apply the Schwarz lemma. So we can conclude that $\left|\Gamma^{\prime}(0)\right| \leq 1$. By the chain rule, $\Gamma^{\prime}(0)=L^{\prime}(k(0)) k^{\prime}(0)=L^{\prime}(-1) k^{\prime}(0)$. Since $L^{\prime}(w)=-2 /(w-1)^{2}$ we get $\Gamma^{\prime}(0)=$ $-2 /(-1-1)^{2} k^{\prime}(0)$ and therefore $k^{\prime}(0)=-2 \Gamma^{\prime}(0)$. Hence we get $\left|k^{\prime}(0)\right| \leq 2$. Since $k(z)=h(z) / a$, we can conclude next that $\left|k^{\prime}(0)\right|=\left|h^{\prime}(0)\right| / a$. Hence $\left|h^{\prime}(0)\right|=a\left|k^{\prime}(0)\right| \leq$ $a \cdot 2$, so $\left|h^{\prime}(0)\right| \leq 2 a$. Next recall that $h(z)=\log f(z)$, so $f(z)=e^{h(z)}$. Hence $f^{\prime}(z)=$ $e^{h(z)} h^{\prime}(z)$. Therefore $f^{\prime}(0)=e^{h(0)} h^{\prime}(0)=f(0) h^{\prime}(0)$. Hence $\left|f^{\prime}(0)\right| \leq|f(0)|\left|h^{\prime}(0)\right|$. This implies that $\left|f^{\prime}(0)\right| \leq 2 a|f(0)|$. Now recall that $\log f(0)=-a$. But we have set this up so that $\log f(0)=\log |f(0)|+i \arg f(0)$ is real-valued. So $\log |f(0)|=-a$, i.e. $\log (1 /|f(0)|)=a$. Therefore $\left|f^{\prime}(0)\right| \leq 2 a|f(0)|=2|f(0)| \log (1 /|f(0)|)$. This concludes the proof of the lemma.

COROLlaRY 1. Suppose that we have two functions $f$ and $g$ holomorphic on the unit disc with $f-g \in H_{1}^{\infty}(\Delta)$. Suppose that $f(z) \neq g(z)$ for each $z \in \Delta$. We then have the estimate $\left|f^{\prime}(z)-g^{\prime}(z)\right| \leq 4|f(z)-g(z)| \log (1 /|f(z)-g(z)|)$ for all $z \in \Delta,|z|<1 / 2$.

Proof. Pick $z,|z|<1 / 2$. We define $G(w)=f(z+w / 2)-g(z+w / 2)$. Then $G(w)$ satisfies the conditions of Lemma 1 . Hence $\left|G^{\prime}(0)\right| \leq 2|G(0)| \log (1 /|G(0)|)$. Therefore,

$$
\frac{1}{2}\left|f^{\prime}(z)-g^{\prime}(z)\right| \leq 2|f(z)-g(z)| \log \frac{1}{|f(z)-g(z)|} .
$$

\section{Approximation for complex curves in $\mathbb{C}^{2}$}

We assume that for every $c=(a, b)=(a+i b) \in \mathbb{C}$ we have a holomorphic graph $\Gamma_{c}$ given by $w=y_{1}+i y_{2}=f_{c}(z), z=x_{1}+i x_{2} \in \Delta$. We assume that all surfaces are disjoint and that there is a surface through every point in $\Delta \times \mathbb{C}$. We assume that $f_{c}(0)=c$.

Let $\pi: \Delta \times \mathbb{C} \rightarrow \mathbb{C}$ be defined by $\pi\left(z, f_{c}(z)\right)=c$. By the discussion in the previous section the function $\pi$ is continuous.

Fix a positive constant $R$. By Corollary 1 there exists a positive real number $\delta_{0}>0$ such that if $z \in(1 / 2) \Delta$ and if $c, c^{\prime} \in R \Delta$ with $\left|c-c^{\prime}\right|<\delta_{0}$ then

$$
\left|\frac{\partial}{\partial z} f_{c^{\prime}}(z)-\frac{\partial}{\partial z} f_{c}(z)\right| \leq 4 \cdot\left|f_{c^{\prime}}(z)-f_{c}(z)\right| \log \frac{1}{\left|f_{c^{\prime}}(z)-f_{c}(z)\right|} .
$$

We define a class of partially-smooth functions:

$$
\begin{aligned}
\mathcal{A}:= & \left\{\phi \in \mathcal{C}(\Delta \times \mathbb{C}): \phi\left(z, f_{c}(z)\right) \in \mathcal{C}^{1}\left(\Gamma_{c}\right),\right. \\
& \Phi\left(x_{1}, x_{2}, w\right):=\frac{\partial}{\partial x_{1}} \phi\left(x_{1}, x_{2}, f_{c}\left(x_{1}, x_{2}\right)\right), w=f_{c}\left(x_{1}, x_{2}\right) \in \mathcal{C}(\Delta \times \mathbb{C}), \\
& \left.\Psi\left(x_{1}, x_{2}, w\right):=\frac{\partial}{\partial x_{2}} \phi\left(x_{1}, x_{2}, f_{c}\left(x_{1}, x_{2}\right)\right), w=f_{c}\left(x_{1}, x_{2}\right) \in \mathcal{C}(\Delta \times \mathbb{C})\right\} .
\end{aligned}
$$


THEOREM 1. Let $\phi \in \mathcal{A}$, let $R$ be a positive real number and let $\epsilon>0$. Then there exists a function $\psi \in \mathcal{C}^{1}(\Delta \times R \Delta)$ such that for every point $\left(x_{1}, x_{2}, w\right)=\left(x_{1}, x_{2}, f_{c}\left(x_{1}, x_{2}\right)\right) \in$ $\Delta \times R \Delta$ :

$$
\begin{gathered}
\left|\psi\left(x_{1}, x_{2}, w\right)-\phi\left(x_{1}, x_{2}, w\right)\right|<\epsilon, \\
\left|\frac{\partial}{\partial x_{1}}\left[\psi\left(x_{1}, x_{2}, f_{c}\left(x_{1}, x_{2}\right)\right)\right]-\frac{\partial}{\partial x_{1}}\left[\phi\left(x_{1}, x_{2}, f_{c}\left(x_{1}, x_{2}\right)\right)\right]\right|<\epsilon, \\
\left|\frac{\partial}{\partial x_{2}}\left[\psi\left(x_{1}, x_{2}, f_{c}\left(x_{1}, x_{2}\right)\right)\right]-\frac{\partial}{\partial x_{2}}\left[\phi\left(x_{1}, x_{2}, f_{c}\left(x_{1}, x_{2}\right)\right)\right]\right|<\epsilon .
\end{gathered}
$$

We will prove the theorem using the following result.

Proposition 1. Let $g \in \mathcal{A}, g\left(x_{1}, x_{2}, f_{a+i b}\left(x_{1}, x_{2}\right)\right)=a$, and let $R$ be a positive real number. There exists a positive real number $t_{0}$ such that the following holds. For all $\epsilon>0$ there exists a function $h \in \mathcal{C}^{1}\left(t_{0} \Delta \times R \Delta\right)$ such that for every point $\left(x_{1}, x_{2}, w\right)=$ $\left(x_{1}, x_{2}, f_{c}\left(x_{1}, x_{2}\right)\right) \in t_{0} \Delta \times R \Delta$ :

$$
\begin{gathered}
\left|h\left(x_{1}, x_{2}, w\right)-g\left(x_{1}, x_{2}, w\right)\right|<\epsilon, \\
\left|\frac{\partial}{\partial x_{1}}\left[h\left(x_{1}, x_{2}, f_{c}\left(x_{1}, x_{2}\right)\right)\right]\right|<\epsilon, \\
\left|\frac{\partial}{\partial x_{2}}\left[h\left(x_{1}, x_{2}, f_{c}\left(x_{1}, x_{2}\right)\right)\right]\right|<\epsilon .
\end{gathered}
$$

The same result holds if we replace $a$ by $b$ in the definition of $g$.

Proof of Theorem 1 from Proposition 1.

LEMMA 2. Let $p \in \Delta$ be a point, and let $R, t_{0}$ be positive real numbers such that $\Delta_{t_{0}}(p) \subset \subset \Delta$. Consider the lamination restricted to $\Delta_{t_{0}}(p) \times \mathbb{C}$. If the conclusion of Proposition 1 holds on $\Delta_{t_{0}}(p) \times R \Delta$ (with respect to projection onto $\{p\} \times \mathbb{C}$ ), then the conclusion of Theorem 1 holds on $\Delta_{t_{0}}(p) \times R \Delta$.

Proof. Let $\pi=\left(\pi_{1}, \pi_{2}\right)$ denote the projection onto $\{p\} \times \mathbb{C}$. For each $j, k \in \mathbb{Z}$ and $\delta>0$ we let $c^{\delta}(j, k)$ denote the point $(p, j \delta+k \delta i)$. Let $\Lambda_{j}^{\delta}$ denote the $\mathcal{C}^{1}$-smooth function defined by $\Lambda_{j}^{\delta}(t)=\cos ^{2}[\pi / 2 \delta(t-j \delta)]$ when $(j-1) \delta \leq t \leq(j+1) \delta$ and 0 otherwise. For each $c^{\delta}(j, k)$ we first define a function

$$
\psi_{j k}^{\delta}(z):=\phi\left(z, f_{c^{\delta}(j, k)}(z)\right),
$$

and then we define a preliminary approximation

$$
\psi^{\delta}(z, w)=\sum_{j, k} \psi_{j k}^{\delta}(z) \Lambda_{j}\left(\pi_{1}(z, w)\right) \Lambda_{k}\left(\pi_{2}(z, w)\right) .
$$

Let $\left(z_{0}, w_{0}\right) \in \Delta_{t_{0}}(p) \times R \Delta$. Then $\pi\left(z_{0}, w_{0}\right)$ is contained in a square with corners $c^{\delta}(j, k), c^{\delta}(j+1, k), c^{\delta}(j, k+1)$ and $c^{\delta}(j+1, k+1)$, and

$$
\psi^{\delta}\left(z_{0}, w_{0}\right)=\sum_{m=j, j+1, n=k, k+1} \psi_{m n}^{\delta}\left(z_{0}\right) \Lambda_{m}\left(\pi_{1}\left(z_{0}, w_{0}\right)\right) \Lambda_{n}\left(\pi_{2}\left(z_{0}, w_{0}\right)\right) .
$$


We have

$$
\begin{aligned}
\left|\psi^{\delta}\left(z_{0}, w_{0}\right)-\phi\left(z_{0}, w_{0}\right)\right|= & \mid \sum_{m=j, j+1, n=k, k+1}\left[\psi_{m n}^{\delta}\left(z_{0}\right)-\phi\left(z_{0}, w_{0}\right)\right] \\
& \times \Lambda_{m}^{\delta}\left(\pi_{1}\left(z_{0}, w_{0}\right)\right) \cdot \Lambda_{n}^{\delta}\left(\pi_{2}\left(z_{0}, w_{0}\right)\right) \mid \\
\leq & \max _{m=j, j+1, n=k, k+1}\left|\psi_{m n}^{\delta}\left(z_{0}\right)-\phi\left(z_{0}, w_{0}\right)\right| .
\end{aligned}
$$

Since the map from $\bar{\Delta}_{t_{0}}(p) \times \mathbb{C}$ defined by $(z, \alpha) \mapsto\left(z, f_{\alpha}(z)\right)$ is a homeomorphism it follows that $\psi^{\delta} \rightarrow \phi$ uniformly as $\delta \rightarrow 0$.

Next we approximate derivatives along leaves. Let $\alpha$ be such that $\left(z_{0}, w_{0}\right)=$ $\left(z_{0}, f_{\alpha}\left(z_{0}\right)\right)$. Since the functions $\Lambda_{j}^{\delta} \circ \pi_{i}$ are constant along leaves,

$$
\begin{aligned}
\mid \frac{\partial}{\partial x_{i}} & {\left[\psi^{\delta}\left(z_{0}, f_{\alpha}\left(z_{0}\right)\right)-\phi\left(z_{0}, f_{\alpha}\left(z_{0}\right)\right)\right] \mid } \\
= & \mid \sum_{m=j, j+1, n=k, k+1}\left[\frac{\partial}{\partial x_{i}}\left[\psi_{m n}^{\delta}\left(z_{0}\right)-\phi\left(z_{0}, f_{\alpha}\left(z_{0}\right)\right)\right]\right] \\
& \times \Lambda_{m}^{\delta}\left(\pi_{1}\left(z_{0}, f_{\alpha}\left(z_{0}\right)\right)\right) \cdot \Lambda_{n}^{\delta}\left(\pi_{2}\left(z_{0}, f_{\alpha}\left(z_{0}\right)\right)\right) \mid \\
\leq & \max _{m=j, j+1, n=k, k+1}\left|\frac{\partial}{\partial x_{i}}\left[\psi_{m n}^{\delta}\left(z_{0}\right)-\phi\left(z_{0}, f_{\alpha}\left(z_{0}\right)\right)\right]\right| .
\end{aligned}
$$

It follows that $\psi^{\delta} \rightarrow \phi$ also in $\mathcal{C}^{1}$-norm on leaves.

Now the conclusion of Lemma 2 follows because the functions $\pi_{j}$ can be approximated uniformly and in $\mathcal{C}^{1}$-norm on leaves.

For each point $p \in \Delta$ there exists by Proposition 1 a positive real number $t_{p}$ such that constant approximation is possible on $\Delta_{t_{p}}(p) \times R \Delta$. Hence by Lemma 2 approximation of functions in $\mathcal{A}$ is possible.

We may then choose a locally-finite cover $\left\{U_{\alpha}\right\}_{\alpha \in \mathbb{N}}$ of $\Delta$ by disks such that approximation by functions in $\mathcal{A}$ is possible on each $U_{\alpha} \times R \triangle$. Let $\left\{\varphi_{\alpha}\right\}$ be a partition of unity subordinate to $\left\{U_{\alpha}\right\}$. For each $\alpha$ let $C_{\alpha}=\left\|\nabla \varphi_{\alpha}\right\|$.

For a given $\epsilon_{\alpha}$ let $g_{\epsilon_{\alpha}}$ be an $\epsilon_{\alpha}$-approximating function of $\phi$ on $U_{\alpha} \times R \triangle$. We will show that there is a sequence $\left\{\epsilon_{\alpha}\right\}$ such that the function

$$
\psi=\sum_{\alpha} \varphi_{\alpha} \cdot g_{\epsilon_{\alpha}}
$$

satisfies the claims of the theorem.

Let $z_{0} \in U_{\alpha}$, and let $\left\{\alpha_{1}, \ldots, \alpha_{m}\right\}$ be the finite set of $\alpha$ 's, such that the support of $\phi_{\alpha}$ intersects $U_{\alpha}$. Then

$$
\psi\left(z, f_{c}(z)\right)=\sum_{i=1}^{m} \varphi_{\alpha_{i}}(z) \cdot g_{\epsilon_{\alpha_{i}}}\left(z, f_{c}(z)\right),
$$


for all $z$ near $z_{0}$. Then

$$
\begin{aligned}
& \left|\psi\left(z_{0}, f_{c}\left(z_{0}\right)\right)-\phi\left(z_{0}, f_{c}\left(z_{0}\right)\right)\right| \\
& \quad=\left|\left[\sum_{i=1}^{m} \varphi_{\alpha_{i}}\left(z_{0}\right) \cdot g_{\epsilon_{\alpha_{i}}}\left(z_{0}, f_{c}\left(z_{0}\right)\right)\right]-\phi\left(z_{0}, f_{c}\left(z_{0}\right)\right)\right| \\
& \quad \leq \sum_{i=1}^{m} \varphi_{\alpha_{i}}\left(z_{0}\right) \cdot\left|g_{\epsilon_{\alpha_{i}}}\left(z_{0}, f_{c}\left(z_{0}\right)\right)-\phi\left(z_{0}, f_{c}\left(z_{0}\right)\right)\right| \\
& \quad \leq \max \left\{\epsilon_{\alpha_{i}}\right\} .
\end{aligned}
$$

Further

$$
\begin{aligned}
\left|\frac{\partial}{\partial x_{1}}\left[\psi\left(z_{0}, f_{c}\left(z_{0}\right)\right)-\phi\left(z_{0}, f_{c}\left(z_{0}\right)\right)\right]\right| \\
=\left|\frac{\partial}{\partial x_{1}}\left[\left[\sum_{i=1}^{m} \varphi_{\alpha_{i}}(z) \cdot g_{\epsilon_{\alpha_{i}}}\left(z_{0}, f_{c}\left(z_{0}\right)\right)\right]-\phi\left(z_{0}, f_{c}\left(z_{0}\right)\right)\right]\right| \\
=\left|\sum_{i=1}^{m} \frac{\partial}{\partial x_{1}}\left[\varphi_{\alpha_{i}}\left(z_{0}\right) \cdot\left(g_{\epsilon_{\alpha_{i}}}\left(z_{0}, f_{c}\left(z_{0}\right)\right)-\phi\left(z_{0}, f\left(z_{0}\right)\right)\right)\right]\right| \\
=\mid \sum_{i=1}^{m} \frac{\partial}{\partial x_{1}}\left[\varphi_{\alpha_{i}}\left(z_{0}\right)\right] \cdot\left(g_{\epsilon_{\alpha_{i}}}\left(z_{0}, f_{c}\left(z_{0}\right)\right)\right)-\left(\phi\left(z_{0}, f\left(z_{0}\right)\right)\right) \\
\quad+\sum_{i=1}^{m} \varphi_{\alpha_{i}}\left(z_{0}\right) \cdot \frac{\partial}{\partial x_{1}}\left[g_{\epsilon_{\alpha_{i}}}\left(z_{0}, f_{c}\left(z_{0}\right)\right)-\phi\left(z_{0}, f\left(z_{0}\right)\right)\right] \mid \\
\leq m \cdot \max \left\{C_{\alpha_{i}}\right\} \cdot \max \left\{\epsilon_{\alpha_{i}}\right\}+\max \left\{\epsilon_{\alpha_{i}}\right\} .
\end{aligned}
$$

Similarly we get that

$$
\left|\frac{\partial}{\partial x_{2}}\left[\psi\left(z_{0}, f_{c}\left(z_{0}\right)\right)-\phi\left(z, f_{c}\left(z_{0}\right)\right)\right]\right| \leq m \cdot \max \left\{C_{\alpha_{i}}\right\} \cdot \max \left\{\epsilon_{\alpha_{i}}\right\}+\max \left\{\epsilon_{\alpha_{i}}\right\} .
$$

It is clear that we may choose $\epsilon_{\alpha_{i}}$ for $i=1, \ldots, m$ to get the desired estimate for all points $z_{0} \in U_{\alpha}$ for this particular $\alpha$. Running through all $\alpha$ we find that any particular $\alpha_{i}$ will only come under consideration a finite number of times. Hence we may choose the sequence $\left\{\epsilon_{\alpha}\right\}$.

We proceed to prove the proposition.

Fix $\delta_{0}$ to get the estimate (1) (in the beginning of $\S 3$ ) for all $\left|c-c^{\prime}\right|<\delta_{0}$ with $|c|,\left|c^{\prime}\right| \leq 2 R$. For any $\delta$ with $0<\delta<\delta_{0}$ we let $c^{\delta}(j, k)=(j+k \cdot i) \cdot \delta$ for $j, k \in \mathbb{Z}$. Let $\chi:[0,1] \rightarrow \mathbb{R}$ be a smooth function such that $\chi(t)=0$ for $0 \leq t \leq 1 / 4$ and $\chi(t)=1$ for $3 / 4 \leq t \leq 1$. Let $C$ be a constant such that $\left|\chi^{\prime}(t)\right| \leq C$ for all $t \in[0,1]$.

We first define a function $h_{\delta}$ on the surfaces $\Gamma_{c^{\delta}(j, k)}$ simply by $\left.h_{\delta}\right|_{\Gamma_{c^{\delta}(j, k)}} \equiv j \delta$. We want to interpolate this function between the surfaces.

For a fixed $z$ consider the sets of points

$$
Q_{c^{\delta}(j, k)}(z):=\left\{f_{c^{\delta}(j, k)}(z), f_{c^{\delta}(j+1, k)}(z), f_{c^{\delta}(j, k+1)}(z), f_{c^{\delta}(j+1, k+1)}(z)\right\} .
$$

We first show that these sets move nicely with $z$ for small enough $|z|$ and independent of $\delta$. In particular we want to know that we may define quadrilateral regions $R_{\delta, j, k}(z)$, with straight edges and corners $Q_{c^{\delta}(j, k)}(z)$, and that these sets have disjoint interior. 
We make the change of coordinates in the $w$ variable, by setting

$$
\tilde{w}(z, w)=\tilde{w}_{j k}(z, w)=\frac{w-f_{c^{\delta}(j, k)}(z)}{f_{c^{\delta}(j+1, k)}-f_{c^{\delta}(j, k)}(z)} .
$$

We get

$$
\begin{gathered}
\tilde{w}\left(z, f_{c^{\delta}(j, k)}(z)\right) \equiv 0, \\
\tilde{w}\left(z, f_{c^{\delta}(j+1, k)}(z)\right) \equiv 1 .
\end{gathered}
$$

From the discussion on holomorphic motions in $§ 2$ we get the following.

Lemma 3. Fix $N$. Then there exists a real number $t_{0}>0$ independent of $\delta$ such that if $|l|,|m|<N$ then $\left|\tilde{w}_{j k}\left(z, f_{c^{\delta}(j+l, k+m)}(z)\right)-\tilde{w}_{j k}\left(z, f_{c^{\delta}(j+l, k+m)}(0)\right)\right|<1 / 10$ for all $|z|<$ $t_{0}$ and any $j, k$.

From now on we assume that $|z| \leq t_{0}$.

LEMMA 4. The quadrilaterals have disjoint interiors.

Proof. Pick $(j, k)$. We use the linear change of coordinates in the $w$ direction for fixed $z$ :

$$
\tilde{w}_{j k}(z, w)=\frac{w-f_{c^{\delta}(j, k)}(z)}{f_{c^{\delta}(j+1, k)}(z)-f_{c^{\delta}(j, k)}(z)} .
$$

This sends $f_{c^{\delta}(j+l, k+m)}(z)$ close to $(j+l, k+m)$ on a small disc in the $z$ direction for uniformly bounded $(l, m)$. Hence it is clear that the quadrilaterals are disjoint.

Next we define preliminary functions $h_{j k}^{\delta}$ on the respective quadrilaterals. First we define a function $t_{z}\left(y_{1}, y_{2}\right)$ to be constant equal to 0 on the line between $f_{c^{\delta}(j, k)}(z)$ and $f_{c^{\delta}(j, k+1)}(z)$, and constant equal to 1 on the line between $f_{c^{\delta}(j+1, k)}(z)$ and $f_{c^{\delta}(j+1, k+1)}(z)$. We extend $t_{z}$ continuously to be affine on the two other edges, and then we extend $t_{z}$ to be constant equal to $v$ on the line between $f_{c^{\delta}(j, k)}(z)+v \cdot\left(f_{c^{\delta}(j+1, k)}(z)-f_{c^{\delta}(j, k)}(z)\right)$ and $f_{c^{\delta}(j, k+1)}(z)+v \cdot\left(f_{c^{\delta}(j+1, k+1)}(z)-f_{c^{\delta}(j, k+1)}(z)\right)$. Finally we define $h_{j k}^{\delta}$ by

$$
h_{j k}^{\delta}\left(z, y_{1}, y_{2}\right)=j \delta+\delta \cdot\left(\chi \circ t_{z}\right)\left(y_{1}, y_{2}\right) .
$$

The $h_{j k}^{\delta}$ patch up smoothly along the vertical sides of the quadrilaterals where the functions are constant. To be able to patch them together in the 'horizontal' directions we first extend each $h_{j k}^{\delta}$ across the 'horizontal' edges.

To do this we use the coordinates defined by $\tilde{w}$. Consider the normalization

$$
\tilde{w}_{j k}(z, w)=\frac{w-f_{c^{\delta}(j, k)}(z)}{f_{c^{\delta}(j+1, k)}(z)-f_{c^{\delta}(j, k)}(z)} .
$$

Let $\tilde{h}_{j k}^{\delta}$ be defined by $\tilde{h}_{j k}^{\delta} \circ \tilde{w}=h_{j k}^{\delta}$. We want to glue together the two functions on the quadrilaterals sharing (in the new coordinates) the line segment $\gamma$ between $(0,0)$ and $(1,0)$, i.e. the function $\tilde{h}_{j k}^{\delta}$ defined above $\gamma$ and the function $\tilde{h}_{j(k-1)}^{\delta}$ below $\gamma$.

We start by extending the function $\tilde{h}_{j k}^{\delta}$. Note first that by Lemma 3 the quadrilaterals $R_{\delta, j, k}$ and $R_{\delta, j, k-1}$ in the new coordinates - henceforth denoted $\tilde{R}_{\delta, j, k}$ and 
$\tilde{R}_{\delta, j, k-1}$ - have corners within (1/10)-distance from the points $(l, m)$ for $l, m \in\{0,1,-1\}$. Note also that if we define a function $\tilde{t}_{z}\left(\tilde{y}_{1}, \tilde{y}_{2}\right)\left(\tilde{w}=\tilde{y}_{1}+i \tilde{y}_{2}\right)$ along lines in the quadrilateral $\tilde{R}_{\delta, j, k}(z)$ in the new coordinates as we did when we defined $t_{z}\left(y_{1}, y_{2}\right)$ above, then $h_{j k}^{\delta}=(j \delta+\delta(\chi \circ \tilde{t})) \circ \tilde{w}$. Because of the placing of the corners we see that there exists a constant $K$ independent of $\delta, j, k$ such that $\left\|\nabla_{\tilde{w}}(j \delta+\delta(\chi \circ \tilde{t}))\right\| \leq K \delta$.

Continue the lines in $\tilde{R}_{\delta, j, k}$ that pass through the interval [(1/8), $\left.1-(1 / 8)\right]$ and extend $\tilde{h}_{j k}^{\delta}$ to be constant on these lines. By the placing of the corners there is a constant $\mu$ - independent of $\delta$ and $j, k$ - such that these lines can be extended to the line between $(0,-\mu)$ and $(1,-\mu)$. Let $\tilde{P}_{\delta, j, k}$ denote the extended set $\tilde{R}_{\delta, j, k} \cup\left(\tilde{R}_{\delta, j, k-1} \cap\left\{y_{2} \geq-\mu\right\}\right)$; we see that $\tilde{h}_{j k}^{\delta}$ extends to be constant on the part of $\tilde{P}_{\delta, j, k}$ where it is not already defined. Extend $\tilde{h}_{j(k-1)}^{\delta}$ similarly in the other direction.

To glue the functions together we choose a smooth function $\varphi\left(z, \tilde{y}_{1}, \tilde{y}_{2}\right)=\varphi\left(\tilde{y}_{2}\right)$ such that $\varphi\left(\tilde{y}_{2}\right)=1$ if $y_{2} \geq \mu$ and such that $\varphi\left(\tilde{y}_{2}\right)=0$ if $y_{2} \leq-\mu$. We define our final function

$$
h_{\delta}(z, w):=\left(\varphi \circ \tilde{w}_{j k}\right)(z, w) \cdot h_{j k}^{\delta}(z, w)+\left(1-\varphi \circ \tilde{w}_{j k}\right)(z, w) \cdot h_{j(k-1)}^{\delta}(z, w) .
$$

Fix a constant $M$ such that $\left\|\partial \varphi / \partial \tilde{y}_{2}\right\|=M$.

LEMMA 5. There are constants $N_{1}$ and $N_{2}$ such that for each $j, k, \delta$ we have $h_{j k}^{\delta}(z, w)=$ $j \delta$ if $\left|w-f_{c^{\delta}(j, k)}(z)\right| \leq N_{1}\left|f_{c^{\delta}(j+1, k)}(z)-f_{c^{\delta}(j, k)}(z)\right|$. Moreover there is a smooth function $\tilde{g}_{j k}^{\delta}\left(z, \tilde{y}_{1}, \tilde{y}_{2}\right)$ such that $h_{j k}^{\delta}=\tilde{g}_{j k}^{\delta} \circ \tilde{w}$ and $\left\|\nabla_{\tilde{w}} \tilde{g}_{j k}^{\delta}\right\| \leq N_{2} \delta$.

Proof. The existence of the constant $N_{1}$ can be seen by our description of the function in local coordinates where we used Lemma 3 . To see the rest let us give the function $\tilde{g}_{j k}^{\delta}$ explicitly.

Fix $z$. Let $\left(a_{1}, a_{2}\right)$ denote the corner of $\tilde{R}_{j, k}^{\delta}$ that is close to $(0,1)$, and define a map $A_{z}\left(\tilde{y}_{1}, \tilde{y}_{2}\right):=\left(\tilde{y}_{1}-\tilde{y}_{2}\left(a_{1} / a_{2}\right), \tilde{y}_{2}\left(1 / a_{2}\right)\right)$. Then $A_{z}$ changes smoothly with $z$ and $\left\|A_{z}\right\|<2$ for all the possibilities of $\left(a_{1}, a_{2}\right)$ we are considering.

Next we define a function $\widehat{t}$ on the quadrilateral $A_{z}\left(\tilde{R}_{j, k}^{\delta}\right)$ along lines as above. Let $\left(b_{1}, b_{2}\right)$ denote the corner close to $(1,1)$ and fix $\widehat{y}=\left(\widehat{y}_{1}, \widehat{y}_{2}\right)$. We have that the two vertical sides of $A_{z}\left(\tilde{R}_{j, k}^{\delta}\right)$ meet at the point $(0,-L)$ where $L=b_{2} /\left(b_{1}-1\right)$. Calculating the slope of the line from the point $\widehat{y}$ to the point $\widehat{t}(\widehat{y}), 0)$, we get that $\widehat{y}_{1} /\left(L+\widehat{y}_{2}\right)=\tilde{t}(y) / L$, which gives us

$$
\widehat{t}(\widehat{y})=\frac{\widehat{y}_{1} \cdot L}{L+\widehat{y}_{2}}=\frac{\widehat{y}_{1} \cdot b_{2}}{b_{2}+\widehat{y}_{2}\left(b_{1}-1\right)} .
$$

We have that $\widehat{t}$ varies smoothly with $\left(b_{1}, b_{2}\right)$ and we see that $\widehat{t}$ has bounded derivatives for the cases of $\left(b_{1}, b_{2}\right)$ we are considering. Define $\tilde{g}_{j k}^{\delta}$ by

$$
\tilde{g}_{j k}^{\delta}=j \delta+\delta\left(\chi \circ \widehat{t} \circ A_{z}\right),
$$

and the function $h_{j k}^{\delta}$ is given by $h_{j k}^{\delta}=\tilde{g}_{j k}^{\delta} \circ \tilde{w}$.

LEMMA 6. $h_{\delta} \rightarrow g$ in sup norm on $\Delta_{t_{0}} \times R \Delta$.

Proof. It is clear that $h_{\delta}(0, \cdot) \rightarrow g(0, \cdot)$ uniformly. The claim then follows from Lemma 8 below. 
LEMMA 7. If $t_{0}$ and $\delta$ are small enough, then $\left|f_{c^{\delta}(j, k)}(z)-f_{c^{\delta}(j+1, k)}(z)\right| \geq \delta^{2}$ for all $z$ with $|z| \leq t_{0}$ and all $j, k$ such that $\left|c^{\delta}(j, k)\right| \leq 2 R$.

Proof. This follows from the Hölder continuity of the holomorphic motion.

Lemma 8. Let $c \in R \bar{\Delta}$. The function $h_{\delta}\left(z, f_{c}(z)\right)$ is small in $\mathcal{C}^{1}$-norm along the graph $\Gamma_{c}$.

Proof. We need to estimate the derivatives of the function $h_{\delta}\left(z, f_{c}(z)\right)$ at an arbitrary point $\left(z_{0}, f_{c}\left(z_{0}\right)\right)$, and this point is contained in some extended quadrilateral $P_{\delta, j, k}$. We estimate $\partial / \partial x=\partial / \partial x_{1}-$ the case of $\partial / \partial x_{2}$ is similar. Since we are working on lines we use the notation $\left(x, y_{1}, y_{2}\right)$ for coordinates.

If the point is close to the vertical edges, then the function $h_{\delta}$ is locally constant, so we are done. We can assume that also $\left(z_{0}, f_{c}\left(z_{0}\right)\right) \in P_{\delta, j, k} \backslash P_{\delta, j, k+1}$. We divide the proof into two cases. Assume first that $\left(z_{0}, f_{c}\left(z_{0}\right)\right)$ is not in $P_{\delta, j, k-1}$. Then the function $h_{\delta}$ is simply equal to the function $h_{j k}^{\delta}$ (see (2)).

We have that

$$
\begin{aligned}
\frac{\partial}{\partial x}\left(h_{j k}^{\delta}(x, f(x))\right) & =\left(\frac{\partial h_{j k}^{\delta}}{\partial x}, \frac{\partial h_{j k}^{\delta}}{\partial y_{1}}, \frac{\partial h_{j k}^{\delta}}{\partial y_{2}}\right)(x, f(x)) \cdot\left(1, \frac{\partial f_{1}}{\partial x}, \frac{\partial f_{2}}{\partial x}\right)(x) \\
& =\frac{\partial h_{j k}^{\delta}}{\partial x}(x, f(x))+\left(\frac{\partial h_{j k}^{\delta}}{\partial y_{1}}, \frac{\partial h_{j k}^{\delta}}{\partial y_{2}}\right)(x, f(x)) \cdot\left(\frac{\partial f_{1}}{\partial x}, \frac{\partial f_{2}}{\partial x}\right)(x) .
\end{aligned}
$$

For fixed $s, v$ we may define a curve $(x, g(x))$ :

$$
\begin{aligned}
g(x)= & (1-s)\left[(1-v) f_{c^{\delta}(j, k)}(x)+v f_{c^{\delta}(j+1, k)}(x)\right] \\
& +s\left[(1-v) f_{c^{\delta}(j, k+1)}(x)+v f_{c^{\delta}(j+1, k+1)}(x)\right] .
\end{aligned}
$$

Then $h_{j k}^{\delta}(x, g(x)) \equiv j \delta+\chi(v) \delta$. Choose $s$ and $v$ so that $\left(x_{0}, g\left(x_{0}\right)\right)=\left(x_{0}, f_{c}\left(x_{0}\right)\right)$. We get that

$$
\begin{aligned}
0 & =\frac{\partial}{\partial x}\left(h_{j k}^{\delta}(x, g(x))\right) \\
& =\frac{\partial h_{j k}^{\delta}}{\partial x}(x, g(x))+\left(\frac{\partial h_{j k}^{\delta}}{\partial y_{1}}, \frac{\partial h_{j k}^{\delta}}{\partial y_{2}}\right)(x, g(x)) \cdot\left(\frac{\partial g_{1}}{\partial x}, \frac{\partial g_{2}}{\partial x}\right)(x),
\end{aligned}
$$

and so substracting (4) from (3) we get

$$
\frac{\partial}{\partial x}\left(h_{j k}^{\delta}\left(x_{0}, f\left(x_{0}\right)\right)\right)=\left(\frac{\partial h_{j k}^{\delta}}{\partial y_{1}}, \frac{\partial h_{j k}^{\delta}}{\partial y_{2}}\right)\left(x_{0}, g\left(x_{0}\right)\right) \cdot\left(\frac{\partial f_{1}}{\partial x}-\frac{\partial g_{1}}{\partial x}, \frac{\partial f_{2}}{\partial x}-\frac{\partial g_{2}}{\partial x}\right)\left(x_{0}\right) \text {. }
$$

Using Lemma 3 we see that $\left\|f_{c}\left(x_{0}\right)-f_{c^{\delta}(j+l, k+m)}\left(x_{0}\right)\right\| \leq 2 \| f_{c^{\delta}(j+1, k)}\left(x_{0}\right)-$ $f_{c^{\delta}(j, k)}\left(x_{0}\right) \|$ for $l, m \in\{0,1\}$, and so 


$$
\begin{aligned}
& \left\|\frac{\partial}{\partial x}\left(f_{c}-f_{c^{\delta}(j+l, k+m)}\right)\left(x_{0}\right)\right\| \\
& \left.\quad \leq 4 \|\left(f_{c}-f_{c^{\delta}(j+l,(k+m)}\right)\left(x_{0}\right)\right) \| \log \frac{1}{\left\|\left(f_{c}-f_{c^{\delta}(j+l, k+m)}\right)\left(x_{0}\right)\right\|} \\
& \quad \leq 8\left\|\left(f_{c^{\delta}(j+1, k)}-f_{c^{\delta}(j, k)}\right)\left(x_{0}\right)\right\| \log \frac{1}{2\left\|\left(f_{c^{\delta}(j+1, k)}-f_{c^{\delta}(j, k)}\right)\left(x_{0}\right)\right\|} .
\end{aligned}
$$

It follows that

$$
\begin{aligned}
\left\|\frac{\partial}{\partial x}\left(h_{\delta}(x, f(x))\right)\right\| \leq & 8 \cdot\left\|\left(\frac{\partial h_{\delta}}{\partial y_{1}}, \frac{\partial h_{\delta}}{\partial y_{2}}\right)\right\| \cdot\left\|\left(f_{c^{\delta}(j+1, k)}-f_{c^{\delta}(j, k)}\right)\left(x_{0}\right)\right\| \\
& \times \log \frac{1}{2\left\|\left(f_{c^{\delta}(j+1, k)}-f_{\delta^{\delta}(j, k)}\right)\left(x_{0}\right)\right\|} .
\end{aligned}
$$

We proceed to estimate $\left\|\left(\partial h_{\delta} / \partial y_{1}, \partial h_{\delta} / \partial y_{2}\right)\right\|$. We change coordinates according to Lemma 5 and write $h_{\delta}$ as a composition $\tilde{g}_{\delta} \circ \tilde{w}(y)$. We get $\left\|D_{w} \tilde{w}\right\|=1 /\left(\| f_{c^{\delta}(j+1, k)}\left(x_{0}\right)-\right.$ $\left.f_{c^{\delta}(j, k)}\left(x_{0}\right) \|\right)$, and we have that $\left\|\nabla_{\tilde{w}} \tilde{g}_{\delta}\right\| \leq N_{2} \delta$. This shows that

$$
\left\|\left(\frac{\partial h_{\delta}}{\partial y_{1}}, \frac{\partial h_{\delta}}{\partial y_{2}}\right)\right\| \leq N_{2} \delta \frac{1}{\left\|f_{c^{\delta}(j+1, k)}\left(x_{0}\right)-f_{c^{\delta}(j, k)}\left(x_{0}\right)\right\|} .
$$

This gives

$$
\left\|\frac{\partial}{\partial x}\left(h_{\delta}(x, f(x))\right)\right\| \leq 8 N_{2} \delta \log \frac{1}{\left\|f_{c^{\delta}(j+1, k)}\left(x_{0}\right)-f_{c^{\delta}(j, k)}\left(x_{0}\right)\right\|} .
$$

We have by Lemma 7 that $\left\|f_{c^{\delta}(j+1, k)}\left(x_{0}\right)-f_{c^{\delta}(j, k)}\left(x_{0}\right)\right\| \geq \delta^{2}$, and so

$$
\left\|\frac{\partial}{\partial x}\left(h_{\delta}\left(x_{0}, f\left(x_{0}\right)\right)\right)\right\| \leq 8 N_{2} \delta \log \frac{1}{2 \delta^{2}} \rightarrow 0 \quad \text { as } \delta \rightarrow 0 .
$$

The other case we have to consider is when $\left(z_{0}, f_{c}\left(z_{0}\right)\right)$ is contained in an overlap where we glued our functions together. In that case we may assume that $\left(z_{0}, f_{c}\left(z_{0}\right)\right)$ is also contained in $P_{j(k-1)}^{\delta}$ (see (2)).

Let $\vec{v}$ denote the vector $\vec{v}=\partial / \partial x\left(x_{0}, f_{c}\left(x_{0}\right)\right)$. We have that

$$
\begin{aligned}
\nabla h_{\delta}\left(x_{0}, f_{c}\left(x_{0}\right)\right) \cdot \vec{v}= & \nabla\left[\varphi \circ \tilde{w} \cdot h_{j k}^{\delta}\right]\left(x_{0}, f_{c}\left(x_{0}\right)\right) \cdot \vec{v} \\
& +\nabla\left[(1-\varphi) \circ \tilde{w} \cdot h_{j(k-1)}^{\delta}\right]\left(x_{0}, f_{c}\left(x_{0}\right)\right) \cdot \vec{v} \\
= & h_{j k}^{\delta}\left(x_{0}, f_{c}\left(x_{0}\right)\right) \cdot \nabla[\varphi \circ \tilde{w}]\left(x, f_{c}\left(x_{0}\right)\right) \cdot \vec{v} \\
& +(\varphi \circ \tilde{w})\left(x_{0}, f_{c}\left(x_{0}\right)\right) \cdot \nabla\left[h_{j k}^{\delta}\right]\left(x_{0}, f_{c}\left(x_{0}\right)\right) \cdot \vec{v} \\
& +h_{j(k-1)}^{\delta}\left(x_{0}, f_{c}\left(x_{0}\right)\right) \cdot \nabla[(1-\varphi) \circ \tilde{w}]\left(x, f_{c}\left(x_{0}\right)\right) \cdot \vec{v} \\
& +((1-\varphi) \circ \tilde{w})\left(x_{0}, f_{c}\left(x_{0}\right)\right) \cdot \nabla\left[h_{j(k-1)}^{\delta}\right]\left(x_{0}, f_{c}\left(x_{0}\right)\right) \cdot \vec{v} .
\end{aligned}
$$

By the above calculations we need not worry about the second and fourth term in this sum so we have to check that

$$
\left(h_{j k}^{\delta}\left(x_{0}, f_{c}\left(x_{0}\right)\right)-h_{j(k-1)}^{\delta}\left(x_{0}, f_{c}\left(x_{0}\right)\right)\right) \cdot \nabla[\varphi \circ \tilde{w}]\left(x_{0}, f_{c}\left(x_{0}\right)\right) \cdot \vec{v} \rightarrow 0
$$

as $\delta \rightarrow 0$. 
First of all we have that $\left|h_{j k}^{\delta}\left(x_{0}, f_{c}\left(x_{0}\right)\right)-h_{j(k-1)}^{\delta}\left(x_{0}, f_{c}\left(x_{0}\right)\right)\right| \leq 2 \delta$. Further, $\mid \nabla[\varphi$ $\circ \tilde{w}]\left(x_{0}, f_{c}\left(x_{0}\right)\right) \cdot \vec{v} \mid \leq M \cdot\left\|D[\tilde{w}]\left(x_{0}, f_{c}\left(x_{0}\right)\right)(\vec{v})\right\|$.

Now

$$
D[\tilde{w}]\left(x_{0}, f_{c}\left(x_{0}\right)\right)(\vec{v})=\frac{\partial}{\partial x}\left[\left(x, \frac{f_{c}(x)-f_{c^{\delta}(j, k)}(x)}{f_{c^{\delta}(j+1, k)}(x)-f_{c^{\delta}(j, k)}(x)}\right)\right]\left(x_{0}\right) .
$$

Ignoring the constant term (it gets killed by $\delta$ ), we get that

$$
\begin{aligned}
\left\|D[\tilde{w}]\left(x_{0}, f_{c}\left(x_{0}\right)\right)(\vec{v})\right\| \leq & \frac{\left|f_{c}^{\prime}\left(x_{0}\right)-f_{c^{\delta}(j, k)}^{\prime}\left(x_{0}\right)\right|}{\left|f_{c^{\delta}(j+1, k)}\left(x_{0}\right)-f_{c^{\delta}(j, k)}\left(x_{0}\right)\right|} \\
& +\frac{\left|f_{c}\left(x_{0}\right)-f_{c^{\delta}(j, k)}\left(x_{0}\right)\right| \cdot\left|f_{c^{\delta}(j+1, k)}^{\prime}\left(x_{0}\right)-f_{c^{\delta}(j, k)}^{\prime}\left(x_{0}\right)\right|}{\left|f_{c^{\delta}(j+1, k)}\left(x_{0}\right)-f_{c^{\delta}(j, k)}\left(x_{0}\right)\right|^{2}} \\
\leq & \frac{\left|f_{c}\left(x_{0}\right)-f_{c^{\delta}(j, k)}\left(x_{0}\right)\right|}{\left|f_{c^{\delta}(j+1, k)}\left(x_{0}\right)-f_{c^{\delta}(j, k)}\left(x_{0}\right)\right|} \log \frac{1}{\left|f_{c}\left(x_{0}\right)-f_{c^{\delta}(j, k)}\left(x_{0}\right)\right|} \\
& +\frac{\left|f_{c}\left(x_{0}\right)-f_{c^{\delta}(j, k)}\left(x_{0}\right)\right| \cdot\left|f_{c^{\delta}(j+1, k)}\left(x_{0}\right)-f_{c^{\delta}(j, k)}\left(x_{0}\right)\right|}{\left|f_{c^{\delta}(j+1, k)}\left(x_{0}\right)-f_{c^{\delta}(j, k)}\left(x_{0}\right)\right|^{2}} \\
& \times \log \frac{1}{\left|f_{c^{\delta}(j+1, k)}\left(x_{0}\right)-f_{c^{\delta}(j, k)}\left(x_{0}\right)\right|} .
\end{aligned}
$$

By Lemma 3, $\left|f_{c}\left(x_{0}\right)-f_{c^{\delta}(j, k)}\left(x_{0}\right)\right| /\left|f_{c^{\delta}(j+1, k)}\left(x_{0}\right)-f_{c^{\delta}(j, k)}\left(x_{0}\right)\right| \leq 2$, and so

$$
\begin{aligned}
\left\|D[\tilde{w}]\left(x_{0}, f_{c}\left(x_{0}\right)\right)(\vec{v})\right\| \leq & 2 \cdot \log \frac{1}{\left|f_{c^{\delta}(j+1, k)}\left(x_{0}\right)-f_{c^{\delta}(j, k)}\left(x_{0}\right)\right|} \\
& +2 \log \frac{1}{\left|f_{c}\left(x_{0}\right)-f_{c^{\delta}(j, k)}\left(x_{0}\right)\right|} .
\end{aligned}
$$

By Lemma 5, our function is constant unless $\left|f_{c}\left(x_{0}\right)-f_{c^{\delta}(j, k)}\left(x_{0}\right)\right| \geq N_{1} \mid f_{c^{\delta}(j+1, k)}\left(x_{0}\right)$ $-f_{c^{\delta}(j, k)}\left(x_{0}\right) \mid \geq N_{1} \delta^{2}$ (by Lemma 7), and so we may assume that

$$
\left\|D[\tilde{w}]\left(x_{0}, f_{c}\left(x_{0}\right)\right)(\vec{v})\right\| \leq 2 \log \frac{1}{\delta^{2}}+2 \log \frac{1}{N_{1} \delta^{2}} .
$$

All in all:

$$
\begin{aligned}
& \left|\left(h_{j k}^{\delta}\left(x_{0}, f_{c}\left(x_{0}\right)\right)-h_{j(k-1)}^{\delta}\left(x_{0}, f_{c}\left(x_{0}\right)\right)\right) \cdot \nabla[\varphi \circ \tilde{w}]\left(x_{0}, f_{c}\left(x_{0}\right)\right) \cdot \vec{v}\right| \\
& \quad \leq 4 M \delta\left(\log \frac{1}{\delta^{2}}+\log \frac{1}{N_{1} \delta^{2}}\right) \rightarrow 0 \quad \text { as } \delta \rightarrow 0 .
\end{aligned}
$$

\section{Proof of the main theorem}

We are ready to prove the main theorem. As pointed out in $\S 2$, by the theorem of Slodkowski $[9,11]$, we can assume that $\mathcal{L}$ is a lamination of $\Delta \times \mathbb{C}$ as in the previous section.

Proof of the main theorem. Suppose that $T$ is a positive closed $(1,1)$-current on $\Delta^{2}(0,1)$, supported on the laminated set $K$ described in the introduction. We assume that $T$ is subordinate to the lamination $\mathcal{L}$ of $K$. Hence there is a positive measure $\mu$ such that 
$T=\int\left[V_{\alpha}\right] d \mu(\alpha)$. Suppose that $\lambda=d w-f_{\alpha}^{\prime}(z) d z$. We want to show that $\lambda \wedge T=0$. Let $\phi$ be any smooth $(1,0)$ test form. We need to show that $\langle\lambda \wedge T, \phi\rangle=0$. This follows since

$$
\begin{aligned}
\langle\lambda \wedge T, \phi\rangle & =\int(\lambda \wedge T) \wedge \phi \\
& =\int T \wedge(\lambda \wedge \phi) \\
& =\int_{\alpha}\left(\int_{V_{\alpha}} \lambda \wedge \phi\right) d \mu(\alpha) \\
& =\int_{\alpha} 0=0 .
\end{aligned}
$$

Assume next that $T$ is directed by $\mathcal{L}$. Since $\mathcal{L}$ is a lamination of $\Delta \times \mathbb{C}$ we may invoke the approximation result from the previous section. With the approximation result at hand the implication follows from Sullivan's proof of the smooth case [10]. We include the proof for the benefit of the reader.

Step 1 is to show that there exists a family of probability measures $\sigma_{\alpha}$ such that $\sigma_{\alpha}$ is supported on $\Gamma_{\alpha}$, and a measure $\mu^{\prime}$ on the $\alpha$-plane such that for all test forms $\omega$,

$$
T(\omega)=\int\left(\int_{\Gamma_{\alpha}} \omega d \sigma_{\alpha}\right) d \mu^{\prime} .
$$

Let $\omega$ be a $(1,1)$ test form and let $\lambda(z, w)=d w-f_{\alpha}^{\prime}(z) d z$ for $w=f_{\alpha}(z)$. Let $\vec{v}_{1}(z, w)=\left(1, f_{\alpha}^{\prime}(z)\right)$ and let $\vec{v}_{2}(z, w)=\left(i, i \cdot f_{\alpha}^{\prime}(z)\right)$ for $w=f_{\alpha}(z)$, and define the 2-tangent field $v(z, w)=\left(\vec{v}_{1}(z, w), \vec{v}_{2}(z, w)\right)$.

Switching basis,

$$
\omega=\psi_{1} d z \wedge d \bar{z}+\psi_{2} d z \wedge \bar{\lambda}+\psi_{3} d \bar{z} \wedge \lambda+\psi_{4} \lambda \wedge \bar{\lambda}
$$

for some functions $\psi_{i}$, and by assumption, $T(\omega)=T\left(\psi_{1} d z \wedge d \bar{z}\right)$. The function $\psi_{1}$ is given by $\psi_{1}=(1 / 2 i) \omega(v)$, and so

$$
T(\omega)=T\left(\frac{1}{2 i} \omega(v) d z \wedge d \bar{z}\right) .
$$

On the other hand we may use $T$ to define a linear functional $L$ on $\mathcal{C}_{0}(\Delta \times \mathbb{C})$ by $L(\psi)=T(\psi d z \wedge d \bar{z})$, and so by the Riesz representation theorem there is a measure $v$ such that

$$
L(\psi)=\int \psi d \nu
$$

This means that

$$
T(\omega)=\int \frac{1}{2 i} \omega(v) d v .
$$

Now the measure $v$ disintegrates [6]: there exists a family of probability measures $\sigma_{\alpha}$ such that $\sigma_{\alpha}$ is supported on $\Gamma_{\alpha}$, and a measure $\mu^{\prime}$ on the $\alpha$-plane such that for all $\psi \in \mathcal{C}_{0}(\Delta \times \mathbb{C})$,

$$
\int \psi d v=\int\left(\int_{\Gamma_{\alpha}} \psi d \sigma_{\alpha}\right) d \mu^{\prime} .
$$


We define currents $T_{\alpha}$ by $T_{\alpha}(\omega)=\int_{\Gamma_{\alpha}}(1 / 2 i) \omega(v) d \sigma_{\alpha}$, and we get that

$$
T(\omega)=\int T_{\alpha}(\omega) d \mu^{\prime}
$$

The next step is to show that $T_{\alpha}$ is closed for $\mu^{\prime}$-almost all $\alpha$. Let $\left\{\omega_{j}\right\}$ be a dense set of $\mathcal{C}^{1}$-smooth $(0,1)$ test forms and fix a $j \in \mathbb{N}$. Let $g$ be a continuous function in the $\alpha$-variable and extend $g$ constantly along leaves. We want to show that

$$
\int g \cdot T_{\alpha}(\partial \omega) d \mu^{\prime}=0
$$

because this would imply that $\partial T_{\alpha}=0$ for $\mu^{\prime}$-almost all $\alpha$ (since $g$ is arbitrary).

By Theorem 1 there exists a sequence $g_{i}$ of smooth functions such that $g_{i} \rightarrow g$ uniformly and in $\mathcal{C}^{1}$-norm on leaves. Since $T$ is closed,

$$
0=\int T_{\alpha}\left(\partial\left(g \omega_{j}\right)\right) d \mu^{\prime}=\int T_{\alpha}\left(\partial g_{i} \wedge \omega_{j}\right) d \mu^{\prime}+\int g_{i} \cdot T_{\alpha}\left(\partial \omega_{j}\right) d \mu^{\prime}
$$

Since $T_{\alpha}\left(\partial g_{i} \wedge \omega\right) \rightarrow 0$ we get that

$$
\int g \cdot T_{\alpha}\left(\partial \omega_{j}\right) d \mu^{\prime}=\lim _{i \rightarrow \infty} \int g_{i} \cdot T_{\alpha}\left(\partial \omega_{j}\right) d \mu^{\prime}=0 .
$$

Running through all $\omega_{j}$ we see that $T_{\alpha}$ is closed for $\mu^{\prime}$-almost all $\alpha$. The only possibility then is that the measures $\sigma_{\alpha}$ are constant multiples of $d z \wedge d \bar{z}$, i.e. $\sigma_{\alpha}=\varphi(\alpha) d z \wedge d \bar{z}$ where $\varphi$ is a measurable function [7]. Define $\mu:=\varphi \cdot \mu^{\prime}$.

\section{Two counterexamples}

In [5] the authors proved versions of the main theorem for real laminations in $\mathbb{R}^{2}$ and $\mathbb{R}^{3}$. In those results we added an extra slope condition on the laminations which is analogous to the estimate in Corollary 1. We give here a simple example of a lamination of curves in $\mathbb{R}^{2}$ where the slope condition is not satisfied. Also, the conclusion of the main theorem fails. The analogue of Theorem 1, i.e. approximation of partially-smooth functions, fails as well.

For each $t \in \mathbb{R}$, we let $\gamma_{t}$ be the curve $y=f_{t}(x)=(x-t)^{3}$ in $\mathbb{R}^{2}$. Clearly this gives a continuous lamination of $\mathbb{R}^{2}$ by curves. The curves are all tangent to the $x$-axis. This implies that the current of integration of the $x$-axis is annihilated by the 1 -form $\lambda$ defined by $d y-f_{t}^{\prime}(x) d x$ on $\gamma_{t}$. However, this current is not an integral of currents $\left[\gamma_{t}\right]$. We also observe that the function $a(x, y)$ defined by $a\left(x, f_{t}(x)\right)=t$ cannot be approximated by $\mathcal{C}^{1}$ functions, because any such approximation will have to have a small derivative along the $x$-axis.

We can also modify this example so that we have a Riemann surface lamination in $\mathbb{C}^{3}$. For $t \in \mathbb{C}$, let $\gamma_{t}$ be the complex curve $\gamma_{t}(s)=(z, w, \tau)=\left(s,(s-t)^{2},(s-t)^{3}\right)$. These curves laminate $\mathbb{C}^{3}$, and $\gamma_{t}$ is tangent to the $z$-axis at $(t, 0,0)$. Hence the $z$-axis is annihilated by any continuous 1 -forms defining the lamination. Hence the current of integration of the $z$-axis is directed. But clearly it is not subordinate to the lamination. Again the function $a(z, w, \tau)$ defined by $\left.a\right|_{\gamma_{t}}=t$ cannot be approximated by $\mathcal{C}^{1}$ functions. 
Acknowledgements. The first author is supported by an NSF grant. The third author is supported by Schweizerische Nationalfonds grant 200021-116165/1.

\section{REFERENCES}

[1] B. Berndtsson and N. Sibony. The $\bar{\partial}$-equation on a positive closed current. Invent. Math. 147 (2002), 371-428.

[2] E. Bedford, M. Lyubich and J. Smillie. Polynomial diffeomorphisms of $\mathbb{C}^{2}$, IV. Invent. Math. 112 (1993), 77-125.

[3] R. Dujardin. Approximation des fonctions lisses sur certaines laminations. Indiana Univ. Math. J. 55 (2006), 579-592.

[4] J. E. Fornæss and N. Sibony. Harmonic currents and finite energy of laminations. Geom. Funct. Anal. 15 (2005), 962-1003.

[5] J. E. Fornæss, Y. Wang and E. F. Wold. Approximation of partially smooth functions. Proceedings of Qikeng Lu Conference (June 2006). Sci. China A: Math. 51 (4) (2008), 553-561.

[6] P. R. Halmos. Measure Theory. Van Nostrand, New York, 1950.

[7] P. Lelong. Fonctions Plurisubharmoniques et Formes Différentielles Positives. Gordon and Breach, Paris, London, New York, 1968.

[8] P. Mañé, P. Sad and D. Sullivan. On the dynamics of rational maps. Ann. Sci. École Norm. Sup. 16 (1983), 193-217.

[9] Z. Słodkowski. Holomorphic motions and polynomial hulls. Proc. Amer. Math. Soc. 111 (1991), 347-355.

[10] D. Sullivan. Cycles for the dynamical study of foliated manifolds and complex manifolds. Invent. Math. 36 (1976), 225-255.

[11] K. Astala and G. J. Martin. Holomorphic motions. Papers on Analysis (A volume dedicated to Olli Martio on the occasion of his 60th birthday). Report, No. 83, University of Jyväskylä, Jyväskylä, 2001, pp. $27-40$. 